\title{
Atom Probe Tomography: Beyond the Microscope, a Breakthrough Backdoor for Chemical, Physical and Functional Characterization at the Nanometer Scale
}

\author{
François Vurpillot ${ }^{1}$, Aurore Gaillard ${ }^{1}$, Laurent Arnoldi ${ }^{1}$, Angela Vella ${ }^{1}$, Lorenzo Rigutti ${ }^{1}$, Vincent
} Tognetti $^{2}$ and Bernard Deconihout ${ }^{1}$

1. Groupe de Physique des Matériaux, UMR CNRS 6634, Université et INSA de Rouen, Saint Etienne du Rouvray, France

2. LCOBS - UMR CNRS 6014, Université de Rouen, Mont Saint Aignan, France

In material science, Atom probe tomography is now considered as a routine tool for the microscopic investigation of phase separations, segregation, clustering, or defects analysis, in 3D and on a nanometer scale. The range of application covers a large panel of materials from metals to insulators. Nevertheless, this technique still presents a certain number of open questions, especially concerning the complex lasermatter interaction mechanisms and the physics of field ion emission under ultrafast laser pulse. The absorption mechanisms in insulators, the relaxation of the absorbed energy following the pulse, the dynamics of surface atoms are some examples of the problems requiring a systematic investigation. Moreover, the laser-matter interaction and the evaporation behavior significantly depend on the shape and composition of the studied object, and this may introduce a certain number of aberrations in the trajectories of evaporated ions, which sometimes limit the accuracy of the reconstruction of the original position of the atoms in the sample. These shortcomings are generally bypassed by a systematic study of the analyzing conditions giving rise to the expected result (or the "best" result) in term of composition and image accuracy.

Looking to this problem from another point of view, these bottlenecks are induced by the complex nature of the mechanisms giving rise to the analysis. The APT data gives a direct or indirect access to these physical mechanisms on a very small surface (from the atomic scale to the end apex scale), a very short timescale (from fs to ns) and under intense stresses (temperature gradient, electric field...). As a result, APT is a technique for nanoscale analysis reaching even beyond the $3 \mathrm{D}$ reconstruction. We will show through different examples that physical measurements may be obtained from the analysis of AP data.

Among these examples, the plasmonic response of metallic tips to the laser illumination is measured accurately through the dynamic of field evaporation from the tip (figure 1)[1]. In semiconductor materials and in oxides, the optical properties under extreme electric field are also emphasized through this dynamic. In addition, new instrumental tools are under development to correlate, in an Atom Probe, the 3D image of atoms produced by the nano-analysing tool to the absorbed or re-emitted light by the tip (figure 2) [2]. Physical properties such as dielectric permittivity and electrical conductivity can also be measured in Atom Probe at the scale of the sample. It can be shown that the electrical conductivity of oxides or semiconductors at low temperature is strongly modified by the surface defects and the presence of charge at the tip surface [3]. More fundamentally, the field evaporation process may be studied directly from the sequence of impacts and from the ion trajectory artifacts that occur in the microscope. A correlation between experiments and modeling approaches brings some new insights of the physics taking place at the tip surface under high electric field [4]. 
References:

[1] L. Arnoldi, A. Vella, J. Houard, and B. Deconihout, Appl. Phys. Lett. 101, (2012) 153101

[2] F. Vurpillot, A. Gaillard, G. Da Costa, B. Deconihout, Ultramicroscopy, (2013), in press

[3] L. Rigutti et al., Ultramicroscopy, (2013), in press

[4] The authors acknowledge funding from the Labex EMC3 (project ASAP funded by French ANR)

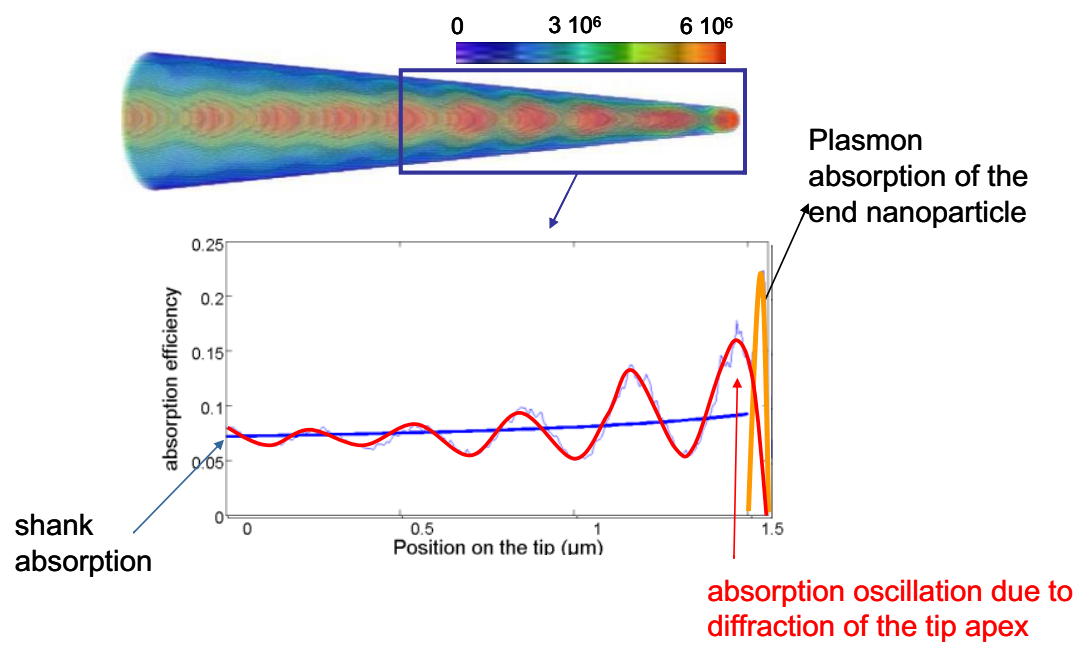

Figure 1. 3D Absorption map of a metallic tip excited by an UV illumination. The temperature dynamic of the tip, in the sub-nanosecond domain is extracted from time-of-flight data in an Atom Probe.

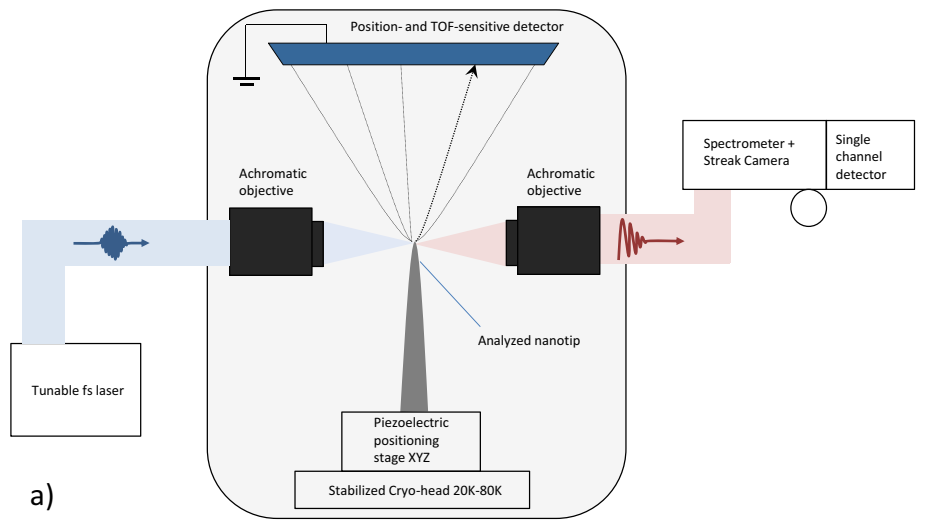

Figure 2. Schemes of an APT instrument coupled with optical spectroscopy in the configuration used for the analysis of the emission of light by Micro Photo Luminescence. 